\title{
Kardiologische Rehabilitation
}

\section{Auch für herzinsuffiziente Patienten sinnvoll?}

\begin{abstract}
Für Koronar-Patienten ist insbesondere nach einem kardiovaskulären Ereignis eine Rehabilitation immer sinnvoll und notwendig, um die medikamentöse Behandlung zu optimieren und nachhaltige Veränderungen des Lebensstils zu erreichen. Gilt dies auch für Patienten mit Herzinsuffizienz? Profitieren auch sie von einer solchen Rehabilitation und wenn ja, prognostisch oder nur hinsichtlich der Lebensqualität?
\end{abstract}

I_a. 2\% der Gesamtbevölkerung haben eine Herzinsuffizienz, wobei die Prognose bei schwerer Herzinsuffizienz durchaus mit der einer malignen Erkrankung vergleichbar ist. Die häufigsten Ursachen einer Herzinsuffizienz sind heute die KHK und die arterielle Hypertonie. Seltener liegt der Erkrankung eine Kardiomyopathie oder ein Klappenvitium zugrunde.

\section{Häufige Begleiterkrankungen}

Nicht bei allen Patienten mit einer Herzinsuffizienz findet sich eine systolische Dysfunktion. „Bei fast jedem zweiten herzinsuffizienten Patienten handelt es sich heute um eine diastolische Herzinsuffizienz", sagte Dr. Hermann Fischer, Dresden. Die diastolische Herzinsuffizienz dürfe keinesfalls als leichtere Form der Herzschwäche angesehen werden, vielmehr sei die Prognose bei beiden Formen der Herzinsuffizienz gleich.

Die Prognose der Herzinsuffizienz wird jedoch nicht nur durch die kardiale Situation bestimmt. Auch die Begleiterkrankungen spielen sowohl im Hinblick auf die Lebensqualität als auch auf die Überlebenszeit eine entscheidende Rolle.,Die häufigsten Begleiterkrankungen bei herzinsuffizienten Patienten sind Diabetes mellitus und Depressionen", so Fischer. Jeder dritte herzinsuffiziente Patient leide an einem Diabetes mellitus, wodurch die Prognose zusätzlich signifikant verschlechtert werde.

Spiroergometrie zur Risikostratifizierung Die alleinige Bestimmung der Auswurffraktion und die Beurteilung hinsichtlich des Schweregrades nach der NYHA-Klassifikation reicht nicht aus, um den funktionellen Status bei herzinsuffizienten Patienten ausreichend zu beschreiben. „In jedem Fall ist die Durchführung einer Spiroergometrie sinnvoll", so Fischer. Damit werde die maximale Sauerstoffaufnahme unter Belastung bestimmt. Dieser Wert spiele auch eine wichtige Rolle bei der Indikationsstellung für die Herztransplantation. Dieses Verfahren könne den Erfolg der medikamentösen und interventionellen Therapie objektivieren und biete zuverlässige Daten für die sozialmedizinische Beurteilung des herzinsuffizienten Patienten.

\section{Prognostische Verbesserung nicht belegt} Daten aus größeren randomisierten kontrollierten Studien, die für die Rehabilitation eine Senkung der Mortalität bei herzinsuffizienten Patienten nachweisen, liegen bisher nicht vor. Auch in der HF-ACTION-Studie, die bei 2131 herzinsuffizienten Patienten durchgeführt wurde, konnte durch ein entsprechendes Trainingsprogramm weder die Gesamtmortalität noch die Notwendigkeit einer erneuten Krankenhausaufnahme signifikant reduziert werden.,Doch Überleben ist nicht alles für diese schwerkranken Patienten", so Fischer. Entscheidend sei auch die Lebensqualität, und diese sei auch in dieser Studie ebenso wie die Symptome und die

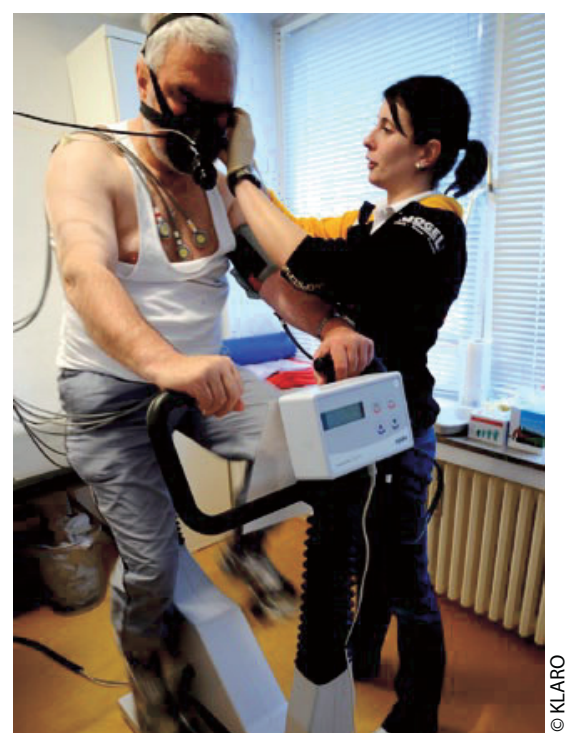

Auch die maximale Sauerstoffaufnahme unter Belastung muss für die Beurteilung des funktionellen Status des Patienten bestimmt werden. soziale Kontextierung durch das Training deutlich verbessert worden.

\section{Welche Rehabilitationsziele?}

Geeignet für eine Rehabilitation sind herzinsuffiziente Patienten im Stadium NYHA I-III. Nach Möglichkeit sollten die Rehabilitationsmaßnahmen, soweit die Infrastruktur es erlaubt, ambulant und nicht stationär durchgeführt werden. Voraussetzung für die Bewegungstherapie ist eine optimale medikamentöse Therapie. Im Vordergrund steht ein Ausdauertraining, was noch durch ein niedrig dosiertes, dynamisches Krafttraining ergänzt werden sollte. Eine weitere wichtige Aufgabe der Rehabilitation sei auch die psychosoziale Beratung und Hilfestellung. Nach Abschluss der Rehabilitationsmaßnahme sollten diese Patienten auch in ein langfristiges Programm, z. B. eine Herzsportgruppe, eingebunden werden. „Die erlernte, individuell dosierte körperliche Trainingstherapie muss anschließend in den Alltag integriert werden", so Fischer.

\section{Wer profitiert am meisten?}

Die Erfolge der Rehabilitationsmaßnahmen sind unabhängig von systolischer oder diastolischer Herzinsuffizienz und der zugrundeliegenden Ursache. „Am meisten profitieren Patienten mit einer Auswurffraktion über $40 \%$ ", so Fischer.

Die strikte Gewichtsreduktion bei übergewichtigen Patienten ist keine evidenzbasierte Empfehlung. Neuere Studienergebnisse zeigen, dass herzinsuffiziente Patienten mit Übergewicht sogar eine bessere Prognose haben als Norm- bzw. Untergewichtige. Kurzum, dicke Kranke leben länger! Auch die häufig empfohlene Salzrestriktion kann nicht mit wissenschaftlichen Daten belegt werden.

"Das eigentliche Ziel der Rehabilitation, auch beim herzinsuffizienten Patienten, müsse sein, ihn zum Spezialisten in seiner eigenen Erkrankung zu machen", so Fischer.

\section{Dr. med. Peter Stiefelhagen II}

II Quelle: 17. Dresdener Symposium „Herz und Gefäße - Innovationen 2011",

19.11.2011 in Dresden 